\title{
The GBA p.G85E mutation in Korean patients with non-neuronopathic Gaucher disease: founder and neuroprotective effects
}

\author{
Yoo-Mi Kim,2, Jin-Ho Choi ${ }^{3}$, Gu-Hwan Kim4, Young Bae Sohn', Jung Min Ko ${ }^{6}$, Beom Hee Lee ${ }^{3,4}$, \\ Chong Kun Cheon ${ }^{7}$, Han Hyuk Lim², Sun-Hee Heo and Han-Wook Yoo ${ }^{3,4^{*}}$ [D
}

\begin{abstract}
Background: Gaucher disease (GD) is caused by a deficiency of $\beta$-glucocerebrosidase, encoded by GBA. Haplotype analyses previously demonstrated founder effects for particular GBA mutations in Ashkenazi Jewish and French-Canadian populations. This study aimed to investigate the clinical characteristics and mutation spectrum of GBA in Korean GD patients and to identify founder effect of GBA p.G85E in non-neuronopathic GD patients.

Results: The study cohort included 62 GD patients from 58 unrelated families. Among them, 18 patients from 17 families harbored the p.G85E mutation. Haplotype analysis was performed for 9 probands and their parents for whom DNA samples were available. In 58 unrelated probands, the GBA mutation p.L483P was the most common (30/116 alleles, 26\%), followed by p.G85E (16\%), p.F252I (13\%), and p.R296Q (9\%). The median age at diagnosis of the 18 patients harboring the p.G85E mutation was 3.8 (range 1.2-57) years. No patients developed neurological symptoms during follow-up periods of 2.2-20.3 (median 13.9) years. The size of the shared haplotype containing GBA p.G85E was $732 \mathrm{kbp}$, leading to an estimated age of 3075 years.
\end{abstract}

Conclusion: The GBA p.G85E mutation, which appears to be neuroprotective despite producing distinctive visceromegaly and skeletal symptoms, exhibited a potential founder effect in Korean GD patients.

Keywords: Gaucher disease, Founder effect, GBA, $\beta$-Glucocerebrosidase

\section{Background}

Gaucher disease (GD [MIM: 230,800, 230,900, and 231,000]) is caused by the deficiency of $\beta$-glucocerebrosidase (GBA), encoded by GBA [1]. Progressive glycolipid accumulation in the reticuloendothelial system leads to anemia, thrombocytopenia, hepatomegaly, splenomegaly, and skeletal manifestations, such as bone pain, avascular necrosis, and osteoporosis $[1,2]$. GD is categorized into non-neuronopathic (type

\footnotetext{
*Correspondence: hwyoo@amc.seoul.kr

${ }^{3}$ Department of Pediatrics, Asan Medical Center Children's Hospital,

University of Ulsan College of Medicine, 88 Olympic-ro 43-gil, Songpa-Gu, Seoul 05505, Korea

Full list of author information is available at the end of the article
}

1), acute neuronopathic (type 2), and chronic neuronopathic (type 3) types, in accordance with the degree of central nervous system involvement [1]. Adult-onset Parkinsonism may occur in patients with type 1 GD $[1,2]$. However, Parkinsonism is not considered as a feature of neuronopathic GD; rather, GBA mutations are one of the risk factors of Parkinsonism [3].

Mutation spectra differ among ethnicities $[4,5]$, and genotype-phenotype correlations have been noted in some mutations. According to the International Collaborative Gaucher Group's Gaucher Registry (clinicaltrials.gov NCT00358943), 94\% of patients in Western countries are of the non-neuronopathic type, whereas half of the patients in Japan, Korea, and China are classified as the neuronopathic type [4]. Common mutations original author(s) and the source, provide a link to the Creative Commons licence, and indicate if changes were made. The images or other third party material in this article are included in the article's Creative Commons licence, unless indicated otherwise in a credit line to the material. If material is not included in the article's Creative Commons licence and your intended use is not permitted by statutory regulation or exceeds the permitted use, you will need to obtain permission directly from the copyright holder. To view a copy of this licence, visit http://creativecommons.org/licenses/by/4.0/. The Creative Commons Public Domain Dedication waiver (http://creativeco mmons.org/publicdomain/zero/1.0/) applies to the data made available in this article, unless otherwise stated in a credit line to the data. 
including c.1226A > G (p.N409S), c.1448 T > C (p.L483P), c.115+1G > A (IVS2+1), and c.84dupG (p.L29Afs"18), account for $90 \%$ of all mutations in the Jewish population and $50 \%$ of all mutations in the non-Jewish populations [3, 6, 7]. Among these, the most prevalent mutation among Caucasians is p.N409S, which accounts for approximately $80 \%$ of mutations [8]. GD patients harboring a p.N409S mutation even in one allele can be excluded from a diagnosis of neuronopathic GD (type 2 or 3) [3], suggesting that the mutation may exert a neuroprotective effect [3, 5-7]. However, the p.N409S mutation has been rarely identified among Asian GD patients, particularly among Japanese and Korean patients [4, 8, 9]. Instead, p.L483P and p.F252I mutations are more prevalent among Asian groups, where the homozygosity of these mutations is generally associated with neuronopathic GD [4, 9-11].

Interestingly, a few recurrent $G B A$ mutations, including p.N409S in Ashkenazi Jewish and European communities [12], p.D448H in northern Swedish [13], p.W417G in French-Canadian [14], and p.G416S in Tabuleiro do Norte, Northeastern Brazilian [15] populations have been suggested as founder mutations.

The GBA p.G85E mutation has been identified in Asian populations, exclusively in Korea, China, and India [4, 9-11, 16]. Furthermore, the p.G85E mutation was found exclusively in non-neuronopathic GD patients [4, 9], suggesting a potential neuroprotective allele. This study aimed to investigate the clinical characteristics of GD patients carrying p.G85E mutation and mutation spectrum of GBA among Korean GD patients and to test for a founder effect for the GBA p.G85E mutation via haplotype analysis.

\section{Results}

\section{Mutation spectrum of Korean GD patients}

Twenty-four different mutations, including 20 missense, one nonsense, one frameshift, one splicing, and recombinants, were identified in 62 patients from 58 unrelated families (Fig. 1a; Table 1). Among the 20 missense variants, 14 pathogenic mutations, and six likely-pathogenic variants (p.V230G, p.P240H, p.R316C, p.D354E, p.N425K, and p.I442T), classified according to American College of Medical Genetics guidelines, were identified.

Thirty-one patients from 30 families had neuronopathic GD, including 10 with type $2(16 \%)$ and 21 with type $3(34 \%)$ GD. Four common mutations identified among Korean GD patients were p.L483P (26\%, 30/116 alleles), p.G85E (16\%, 19/116 alleles), p.F252I (13\%, 15/116 alleles), and p.R296Q (9\%, 10/116 alleles).

p.L483P was the most prevalent mutation in the 30 unrelated neuronopathic GD patients, including two homozygous for p.L483P and 11 compound heterozygotes (25\%, 15/60 alleles) (Fig. 1a). Sixteen different pathogenic mutations were identified in neuronopathic GD patients (Fig. 1b).

In 31 non-neuronopathic GD patients (50\%) from 28 Korean families, 15 different pathogenic mutations were identified, with p.G85E being the most common (34\%, 19/56 alleles) (Fig. 1a; Table 1). This mutation is classified as a pathogenic variant in Clinvar (https://www.ncbi.nlm. nih.gov/clinvar/variation/4296). The allele frequency of p.G85E was 0.000004 in the Genome Aggregation Database (https://gnomad.broadinstitute.org/).

All patients harboring a p.G85E mutation were diagnosed with non-neuronopathic GD (Fig. 1a, b). Among 18 GD patients from 17 unrelated families harboring the p.G85E mutation, two unrelated patients were homozygous for p.G85E and 16 (89\%) were compound heterozygotes harboring GBA mutations associated with neuronopathic phenotype (p.R159W, p.V230G, p.F252I, p.R296Q, p.D438N, p.L483P, and Rec8a) (Table 2). The $\beta$-glucocerebrosidase activity of mutant GBA p.G85E in COS-7 cells was lower $(35 \pm 21 \%)$ than that of wild-type GBA (Fig. 2).

\section{Three-dimensional (3D) structure of GBA and location of mutations}

We determined the location of each mutation in the $3 \mathrm{D}$ structure of GBA (Additional file 1: Figure S1). All mutations were widely located in domains I, II, and III. The p.G85E, p.R87W, p.L483P, and R535H mutations were located in the immunoglobulin (Ig)-like domain (domain II), but they were distant from the active site, except for p.L483P, which was located in the hydrophobic core of the Ig-like domain, which may lead to disruption of the hydrophobic core and alteration of the domain folding. p.G85E, p.R87W, and R535H were identified only in type 1 GD patients. p.V54L, p.N425K, p.D438N, p.I442T, and p.D448H were located in domain I containing two disulphide bridges. p.V54, D438N, and p.I442T were identified in type $1 \mathrm{GD}$. The other 12 missense mutations were located in the catalytic domain, which is the triosephosphate isomerase (TIM)-barrel (domain III). Among mutations located in domain III, p.V211fs and p.W420* were identified in patients with the neuronopathic type. The splicing mutation (IVS2 $+1 \mathrm{G}>\mathrm{A}$ ), which leads to disruption of domains I and II, was also found in type 2 GD patients.

\section{Clinical characteristics of patients harboring the p.G85E mutation}

Eighteen patients with type 1 GD (8 males and 10 females) harbored the p.G85E mutation in at least one allele. The median age at diagnosis was 3.8 (range $1.3-57)$ years. Fourteen (78\%) were diagnosed before 


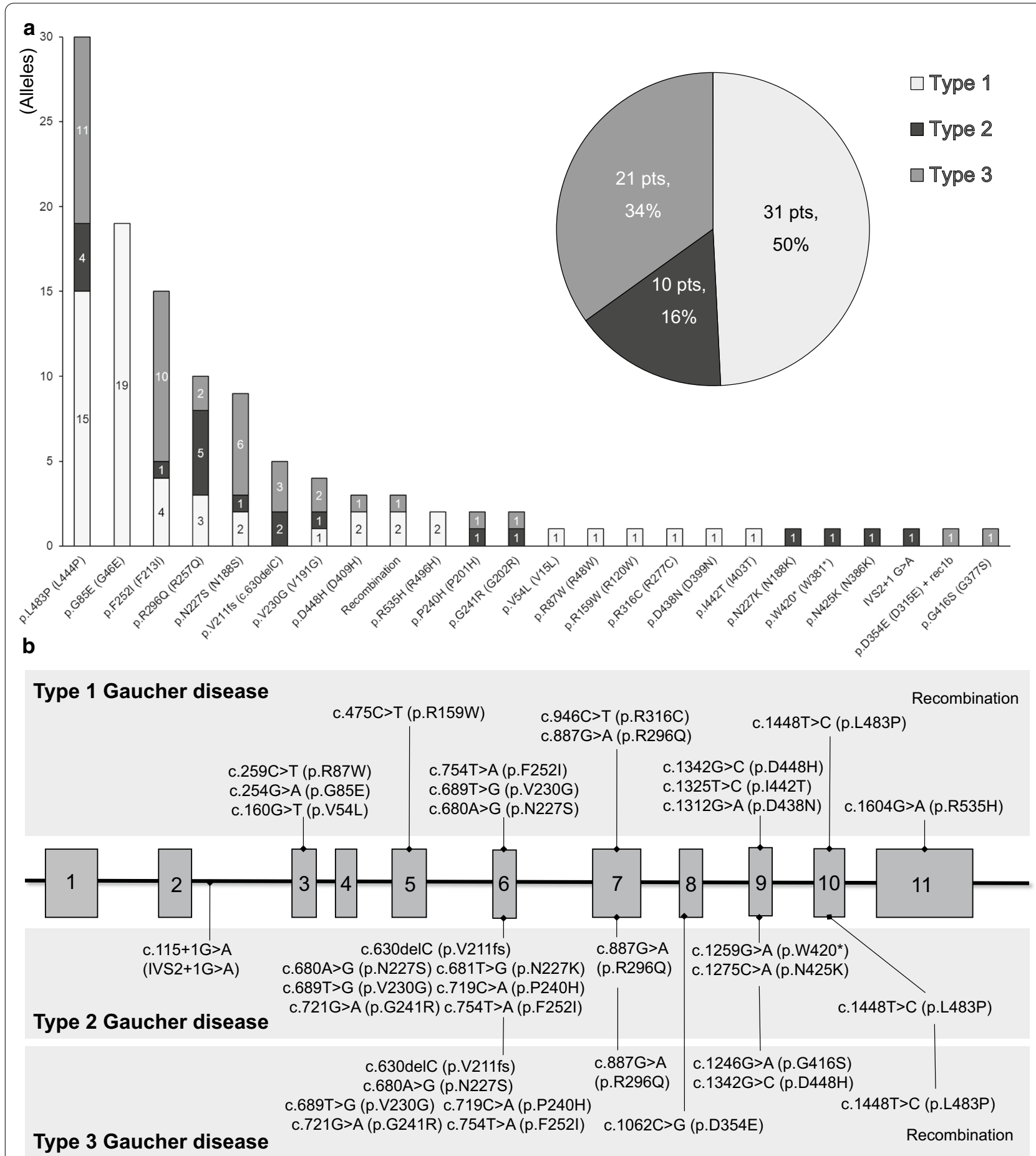

Fig. 1 Proportion of type 1,2 and 3 Gaucher disease and genotype in 62 Gaucher Disease patients from 58 unrelated Korean families (a) and distribution of 24 GBA mutations according to phenotype (b)

18 years of age (Table 2). Their height and weight standard deviation scores (SDS) at diagnosis were $-1.4 \pm 0.8$ and $-1.1 \pm 0.8$, respectively. Two patients (Subjects 16 and 17) homozygous for p.G85E were diagnosed at
7 and 31 years of age, respectively. Other compound heterozygote patients harboring the p.G85E mutation were diagnosed at $9.6 \pm 14.4$ (median 2.9, range 1.3-57) years. 
Table 1 GBA genotypes of 62 Korean Gaucher disease patients from 58 unrelated families

\begin{tabular}{|c|c|c|c|c|c|c|c|c|c|}
\hline Subject & Sex & Type & Allele 1 & Allele 2 & Subject & Sex & Type & Allele 1 & Allele 2 \\
\hline 1 & $\mathrm{~F}$ & 1 & p.G85E (exon 3) & p.R159W (exon 5) & 32 & $M$ & $\|$ & p.N425K (exon 9) & p.L483P (exon 10) \\
\hline 2 & $\mathrm{~F}$ & 1 & p.G85E (exon 3) & p.R296Q (exon 7) & 33 & $\mathrm{~F}$ & $\|$ & p.F252I (exon 6) & p.P240H (exon 6) \\
\hline 3 & $F$ & 1 & p.G85E (exon 3) & p.R296Q (exon 7) & 34 & $F$ & $\|$ & p.W420* (exon 9) & p.L483P (exon 10) \\
\hline 4 & $\mathrm{~F}$ & 1 & p.G85E (exon 3) & Rec8a & 35 & $M$ & $\|$ & p.N227S (exon 6) & p.N227K (exon 6) \\
\hline 5 & $M$ & 1 & p.G85E (exon 3) & p.D438N (exon 9) & 36 & $M$ & $\|$ & p.V211fs (exon 6) & p.R296Q (exon 7) \\
\hline 6 & $M$ & 1 & p.G85E (exon 3) & p.F252l (exon 6) & 37 & $M$ & $\|$ & p.L483P (exon 10) & p.V211fs (exon 6) \\
\hline $7^{a}$ & $\mathrm{~F}$ & 1 & p.G85E (exon 3) & p.F252l (exon 6) & 38 & $M$ & $\|$ & p.R296Q (exon 7) & p.R296Q (exon 7) \\
\hline $8^{a}$ & $M$ & 1 & p.G85E (exon 3) & p.F252l (exon 6) & 39 & $F$ & $\|$ & p.L483P (exon 10) & IVS2 $+1 G>A$ \\
\hline 9 & $M$ & 1 & p.G85E (exon 3) & p.F252I (exon 6) & 40 & $M$ & $\|$ & p.G241R (exon 6) & p.R296Q (exon 7) \\
\hline 10 & $\mathrm{~F}$ & 1 & p.G85E (exon 3) & p.L483P (exon 10) & 41 & $M$ & $\|$ & p.V230G (exon 6) & p.R296Q (exon 7) \\
\hline 11 & $F$ & 1 & p.G85E (exon 3) & p.L483P (exon 10) & 42 & $F$ & III & p.V211fs (exon 6) & p.N227s (exon 6) \\
\hline 12 & M & 1 & p.G85E (exon 3) & p.L483P (exon 10) & 43 & $F$ & III & p.N227S (exon 6) & p.V211fs (exon 6) \\
\hline 13 & $\mathrm{~F}$ & I & p.G85E (exon 3) & p.L483P (exon 10) & 44 & $F$ & III & p.V230G (exon 6) & p.L483P (exon 10) \\
\hline 14 & $M$ & 1 & p.G85E (exon 3) & p.L483P (exon 10) & 45 & $M$ & III & p.L483P (exon 10) & p.L483P (exon 10) \\
\hline 15 & $\mathrm{~F}$ & 1 & p.G85E (exon 3) & p.1442T (exon9) & 46 & $F$ & III & p.F252I (exon 6) & p.L483P (exon 10) \\
\hline 16 & $M$ & 1 & p.G85E (exon 3) & p.G85E (exon 3) & 47 & $M$ & III & p.V211fs (exon 6) & p.N227S (exon 6) \\
\hline 17 & $\mathrm{~F}$ & 1 & p.G85E (exon 3) & p.G85E (exon 3) & 48 & $F$ & III & p.L483P (exon 10) & p.V230G (exon 6) \\
\hline 18 & $M$ & 1 & p.G85E (exon 3) & p.V230G (exon 6) & 49 & $\mathrm{~F}$ & III & p.F252I (exon 6) & p.R296Q (exon 7) \\
\hline 19 & $M$ & 1 & p.V54L (exon3) & p.L483P (exon 10) & 50 & $M$ & III & p.F252I (exon 6) & p.F252I (exon 6) \\
\hline $20^{b}$ & $M$ & 1 & p.R87W (exon 3) & p.R296Q (exon 7) & 51 & $M$ & III & p.F252I (exon 6) & p.L483P (exon 10) \\
\hline $21^{b}$ & $M$ & 1 & p.R87W (exon 3) & p.R296Q (exon 7) & 52 & $F$ & III & p.F252I (exon 6) & p.G416S (exon 9) \\
\hline 22 & $\mathrm{~F}$ & 1 & p.N227S (exon 6) & p.L483P (exon 10) & $53^{d}$ & $F$ & III & p.N227S (exon 6) & p.R296Q (exon 7) \\
\hline 23 & $M$ & 1 & p.N227S (exon 6) & p.L483P (exon 10) & $54^{d}$ & $F$ & III & p.N227S (exon 6) & p.R296Q (exon 7) \\
\hline 24 & $\mathrm{~F}$ & 1 & p.F252I (exon 6) & p.L483P (exon 10) & 55 & $M$ & III & p.F252I (exon 6) & p.F252I (exon 6) \\
\hline 25 & $\mathrm{~F}$ & 1 & p.R316C (exon 9) & p.L483P (exon 10) & 56 & $\mathrm{~F}$ & III & p.P240H (exon 6) & p.L483P (exon 10) \\
\hline $26^{c}$ & $M$ & 1 & p.D448H (exon 9) & p.L483P (exon 10) & 57 & $M$ & III & p.L483P (exon 10) & p.L483P (exon 10) \\
\hline $27^{c}$ & $M$ & 1 & p.D448H (exon 9) & p.L483P (exon 10) & 58 & $F$ & III & p.G241R (exon 6) & p.N227s (exon 6) \\
\hline 28 & $\mathrm{~F}$ & 1 & p.D448H (exon 9) & p.L483P (exon 10) & 59 & $M$ & III & p.D448H (exon 9) & p.L483P (exon 10) \\
\hline 29 & $\mathrm{~F}$ & 1 & p.R535H (exon 11) & p.L483P (exon 10) & 60 & $M$ & III & p.F252I (exon 6) & p.D354E (exon8) + Rec1b \\
\hline 30 & $\mathrm{~F}$ & 1 & p.L483P (exon 10) & p.L483P (exon 10) & 61 & $\mathrm{~F}$ & III & p.N227S (exon 6) & $\operatorname{Rec} 5 b$ \\
\hline 31 & M & 1 & p.R535H (exon 11) & Recla & 62 & M & III & p.L483P (exon 10) & p.F252I (exon 6) \\
\hline
\end{tabular}

$a, b, c, d$ Familial cases

Anemia and thrombocytopenia were assessed upon presentation. Initial hemoglobin and platelet levels were $10.7 \pm 2.1 \mathrm{~g} / \mathrm{dL}$ and $116 \pm 125.9 \times 10^{3} / \mu \mathrm{L}$, respectively; however, 2 patients harboring homozygous p.G85E mutations were not anemic (Table 2).

Four patients (23\%) underwent splenectomy during childhood, prior to the introduction of enzyme replacement therapy (ERT). Of the 4 splenectomized patients (Subjects 7, 12, 15, and 16), two (Subjects 7 and 16) manifested avascular necrosis (AVN) of the femur neck, while Subject 12 had osteoporosis, with a lumbar spine Z-score of -5.4 . Subject 15 had compression fractures in the thoracic spine at age 13 years. Four patients (Subjects 7, 9, 14, and 16) manifested AVN of the femur neck at ages $11,12,12$, and 57 years, respectively. A 2-year-old female patient (Subject 11) suffered from severe bone pain and massive splenomegaly, with abnormally low T1 signal intensity in the long bone and femur neck on magnetic resonance imaging.

Following the introduction of ERT in Korea in 1994, all patients have been treated with it. Patient age at the last evaluation ranged from 3.7 to 67 years (mean $25.7 \pm 17.0$, median 19.0). No patients developed neurological symptoms, including abnormal eye movements, ataxia, seizures, and cognitive impairment during the follow-up period, which ranged from 2.3 to 21 years. After ERT treatment for 2.2-20.3 years (median 13.9), hematological findings improved among all patients, with mean hemoglobin and platelet levels of $13.6 \pm 1.57 \mathrm{~g} / \mathrm{dL}$ and $240 \pm 81.2 \times 10^{3} / \mu \mathrm{L}$, 


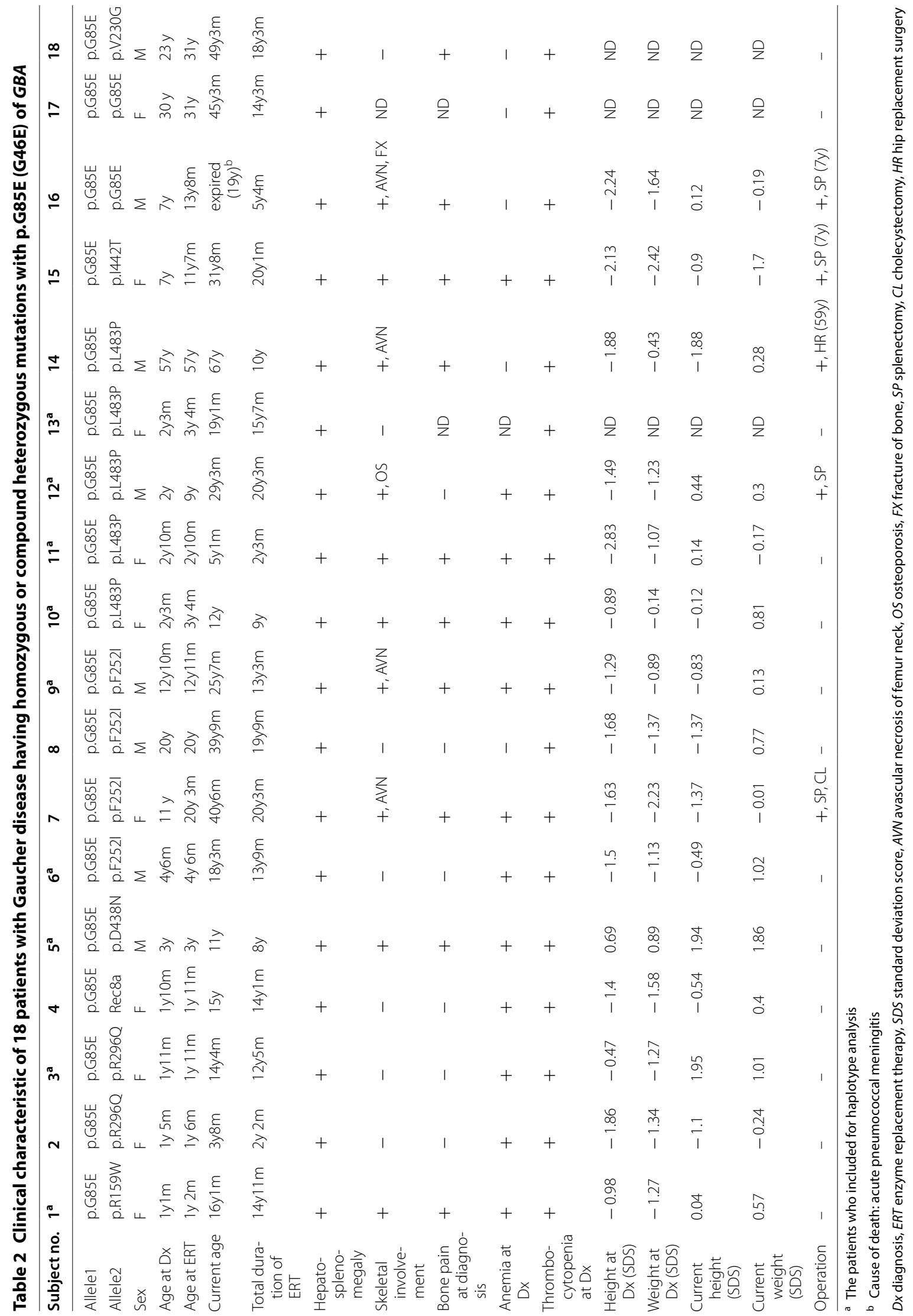




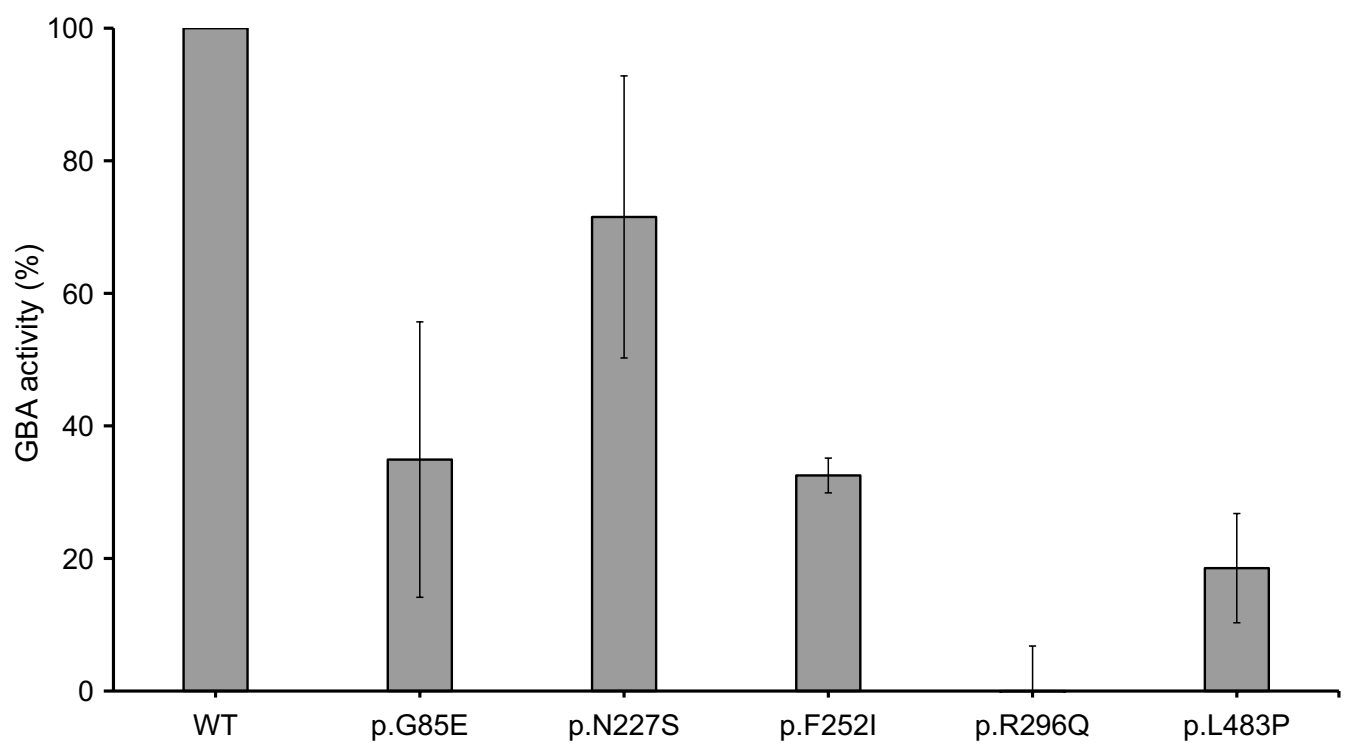

Fig. 2 Comparison of percentages of glucocerebrosidase (GBA) activity in COS-7 cells transiently transfected with wild-type and mutant constructs

respectively. Skeletal complications, including bone pain, AVN, and low bone density, had ameliorated. Symptoms of 10 patients who had complained of bone pain at diagnosis were either decreased or obliterated. Only one patient (Subject 14) with AVN finally underwent total hip replacement surgery at the age of 59 years. The bone mineral density Z-score of Subject 12 improved from -5.4 to -2.4 . Height SDS and weight SDS significantly improved from $-1.4 \pm 0.8$ to $-0.3 \pm 1.3$ and from $-1.1 \pm 0.8$ to $0.1 \pm 0.8$, respectively $(p=0.001)$. Among patients receiving splenectomy prior to ERT, Subject 7 underwent cholecystectomy owing to acute cholecystitis and Subject 16 died of acute bacterial meningitis at age 19 years (Table 2).

\section{Founder effect of the p.G85E mutation among Korean patients}

On haplotype analysis, 9 patients were compound heterozygotes for the p.G85E allele with other mutant GBA alleles. The size of the shared haplotype was approximately $732 \mathrm{kbp}$ (chromosome 1: 154,808,287155,540,660) (Fig. 3) (Additional file 2: Table S1). All 9 alleles containing the p.G85E mutation shared a common haplotype surrounding it, suggesting a founder effect. The estimated age of the mutation was 123 generations (95\% credible set; 69-248 generations); i.e., 3075 years ( $95 \%$ credible set: $1725-6200$ years), based on an intergeneration time of 25 years [17].

\section{Discussion}

This study assessed the mutational spectrum and phenotypic characteristics of Korean GD patients, many of whom share a unique founder mutation p.G85E. In 1996, this mutation was first reported in two Korean patients: 2- and 18-year-old boys, who presented with massive hepatosplenomegaly and isolated splenomegaly, respectively [11]. Both were non-neuronopathic and their genotypes were compound heterozygous for p.L483P and homozygous for p.G85E, respectively [11]. GBA expression of the p.G85E mutant was as low as the activity of the p.F252I mutant in neuronopathic forms [4]. The 3D structure showed that p.G85E was located in the Ig-like domain II (Additional file 1: Figure S1) and distant from the active site residues E274-E379 [18]. In our study, patients with p.G85E presented with massive visceromegaly and bone involvement during early childhood. However, none had shown any neurologic symptoms during a relatively long follow-up period.

Genotype-phenotype correlations have been previously reported, although they have not always been consistent. Patients with at least one p.N409S allele are non-neuronopathic in Caucasians [19-23]. Furthermore, homozygous p.K118N, p.N227S, and p.G416S mutations are also non-neuronopathic. However, compound heterozygosity with null mutant alleles results in the chronic neuronopathic form (type 3) [11, 24-27].

Half of the chronic non-neuronopathic type GD (type 3) patients displayed symptoms before 2 years of age, while others exhibited initial symptoms during 


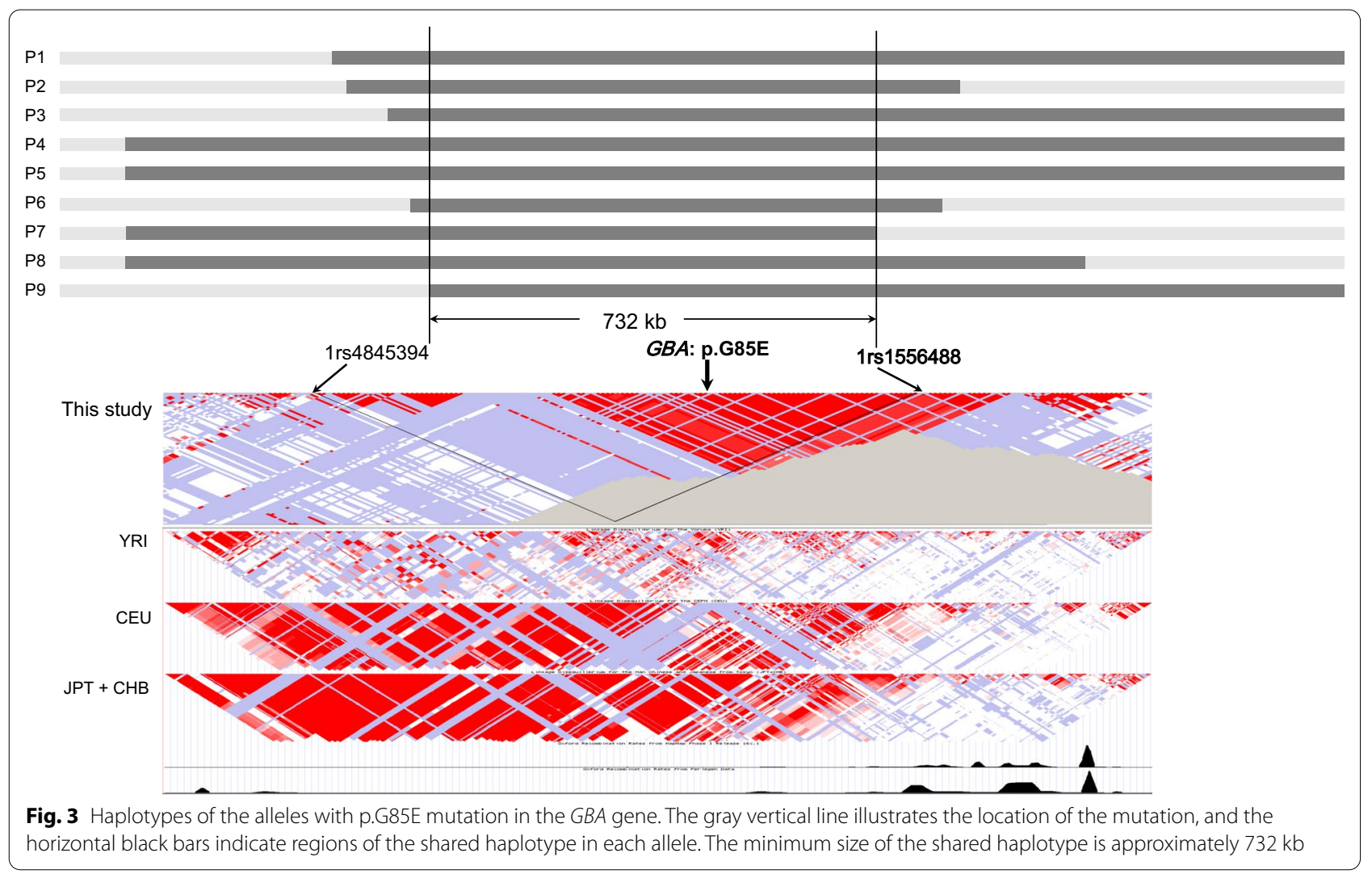

late adolescence [22, 28-30]. In the present study, GD patients carrying the p.G85E mutation have yet to present with any neurologic symptoms at the latest evaluation. More than half of the patients $(11 / 18,61 \%)$ were adults. Furthermore, Subject 4, displaying compound heterozygosity for p.G85E and Rec8a, displayed no neurological symptoms until the latest follow-up at 15 years of age. However, many GD patients with the p.G85E mutation presented with more severe hematological, visceral, and skeletal manifestations, than did typical GD patients.

These findings suggest that the p.G85E mutation may exert a neuroprotective effect, as does the p.N409S mutation in Caucasian GD patients. The p.G85E mutation was previously identified in two Chinese patients as compound heterozygotes, with one having p.N227S and the other having p.L483P, respectively [10]. The patients harboring the p.G85E and p.L483P mutations were diagnosed with type 1 , whereas the other patient with p.G85E and p.N227S was diagnosed with type $3 \mathrm{GD}$; this latter patient presented with seizures and abnormal electroencephalography findings, but not oculomotor abnormalities. Recently, two Indian GD patients with p.G85E mutation were reported, but without phenotypic information [16].
In addition to having unique clinical characteristics, the p.G85E mutation is frequently observed among Korean GD patients, accounting for $16 \%$ of mutant alleles, second only to the p.L483P mutation (26\%). We assessed the haplotypes of alleles with p.G85E and identified a haplotype $732 \mathrm{kbp}$ in length, suggesting that a founder mutation occurred 3,075 years ago. To date, we have not encountered any case of Parkinson disease or Alzheimer disease in a $G B A$ p.G85E carrier, and there have been no reports about the association between GBA p.G85E carriers and Parkinson disease [31]. However, we carefully counseled the patients, together with their parents, considering the late onset of Parkinson disease or Alzheimer disease [32]. Thus, we hypothesize that the p.G85E mutation may have passed through many generations within the Korean population, with positive selection for the neuroprotective effects conferred to carriers.

There are several studies on Korean founder mutations in other monogenic disorders, including p.Q258* of STAR in congenital lipoid adrenal hyperplasia [MIM \#201710] [33], p.P240L of $C D H 23$ in non-syndromic hearing loss [MIM \#601386] [34], and two intronic mutations of UNC13D (c.118-308C > T,c.754-1G >C) in type 3 familial hemophagocytic lymphohistiocytosis [MIM \#608898] [35]. Due to their adjacent geographic locations, Korean, 
Japanese, and Chinese populations often share common mutations contributing to many autosomal recessive disorders, including c.648G > T of G6PC in glycogen storage disease type I [MIM \#232200] [36] and p.R778L of $A T P 7 B$ in Wilson disease [MIM \#277900] [37]. Interestingly, the p.G85E mutation is predominantly found in Korean GD patients, when considering few reports of other Asian countries, such as China and India.

\section{Conclusion}

In conclusion, this study shows the mutation spectrum of $G B A$, as well as clinical features and a founder effect of GBA p.G85E in the Korean population. Our data and those from a previous study [4] strongly suggest that the p.G85E mutation may behave as a neuroprotective allele in GD patients, even though their visceral and skeletal phenotypes might be severe. Further long-term, meticulous clinical follow-up may be needed to understand the neuroprotective effect of p.G85E allele and to elucidate the mechanisms underlying this effect.

\section{Methods}

\section{Patients}

The study cohort included 62 patients with GD from 58 unrelated Korean families diagnosed through molecular analysis of GBA in Korea from 2000 to 2019. Among them, 18 patients from 17 families harbored the p.G85E mutation (Table 2). Their clinical characteristics, including height, weight, visceromegaly, skeletal findings, hematological parameters, bone mineral density assessed through dual-energy X-ray absorptiometry, and neurologic symptoms, were retrospectively reviewed. This study was approved by the Institutional Research Board at each institute. Informed consent was obtained from all patients and their parents.

\section{Molecular analysis of the GBA gene}

Genomic DNA was extracted from peripheral blood leukocytes using the Puregene DNA isolation kit (Qiagen, Hilden, Germany). Polymerase chain reaction (PCR) amplification of all coding exons and exon-intron boundaries was performed using allele-specific primers. Direct bidirectional sequencing of amplification products was performed using an ABI3130xl Genetic Analyzer (Applied Biosystems, Foster City, CA, USA). The longrange PCR approach was used for identification of $G B A$ recombinant mutations between $G B A$ and the non-functional GBAP pseudogene located $16 \mathrm{~kb}$ downstream of the functional $G B A$ according to a previous report [38]. This report uses $G B A$ nomenclature based on the reference sequence NP_000148.2. Traditional GBA nomenclature, which omits the first 39 amino-acid residues of the leader sequence of NP_000148.2, is also provided within parentheses without the p. prefix in Fig. 1.

\section{D structure modeling of GBA}

We generated the 3D structure of GBA using the Cn3D Software 4.3 in National Center for Biotechnology Information (NCBI, https://www.ncbi.nlm.nih.gov/Structure/ $\mathrm{CN} 3 \mathrm{D} / \mathrm{cn} 3 \mathrm{~d}$.shtml) and the mature protein data were obtained from protein data bank with accession code 2NT1-A (https://www.ncbi.nlm.nih.gov/protein/12292 1009) (Additional file 1: Figure S1).

\section{In vitro expression of GBA mutant proteins}

The $\beta$-glucocerebrosidase activity of the p.G85E, p.N227S, p.F252I, p.R296Q, and p.L483P mutant proteins was measured in COS-7 cells transfected with the pCMV6 vector. For site-directed mutagenesis, human GBA (NM_000157.4) cDNA (SC120080 OriGene, Rockville, MD, USA) was used as a template for the generation of mutants. $G B A$ mutants, with a wild-type control, were transiently transfected into COS-7 cells using Effectene transfection reagent \#301427 (Qiagen, Hilden, Germany). After transient transfection, all cells were further incubated at $37^{\circ} \mathrm{C}$ in $5 \% \mathrm{CO}_{2}$. Assays for $\beta$-glucocerebrosidase activity were performed using a standard fluorometric method. Fluorescence was detected using a fluorescence spectrophotometer (Molecular Devices, San Jose, CA, USA). Every experiment was performed in triplicate for each mutant.

\section{Haplotype analysis}

Analysis was performed for 9 unrelated probands and their 16 family members from whom DNA samples were available and who consented to participate in the study. Genotyping of short tandem repeat (STR) and singlenucleotide polymorphism (SNP) markers was performed within the 3.8-Mb genomic region encompassing at least three recombination hotspots on both sides of the mutation [chromosome 1:153,067,786-156,925,007 (hg19)]. Eleven STR markers were selected from the UniSTS database (https://www.ncbi.nlm.nih.gov/probe/?term=Unist s, Additional file 3: Table S2). PCR amplification was performed under standard conditions in a total volume of $50 \mu \mathrm{L}$ containing $50 \mathrm{ng}$ of genomic DNA. Forward primers were labeled with fluorescein amidite. The fluorescently labeled PCR products were sized by comparison to size markers following capillary electrophoresis in an ABI3130xl Genetic Analyzer (Applied Biosystems, Foster City, CA, USA). Data were analyzed using GeneMarker software, version 2.4.1 (SoftGenetics, State College, PA, USA). SNP marker analysis was performed using an Illumina Infinium ${ }^{\circledR}$ Human Omni2.5-8 v1.3 BeadChip (Illumina, San Diego, CA, USA) including 2,372,784 SNP 
markers. Of these, 873 SNP markers within the designated genomic region were selected for analysis.

\section{Estimation of mutation age}

The DMLE2.3 program (https://www.dmle.org/) was used to estimate the age of the GBA p.G85E mutation. The population growth rate was set as 0.025 , with an intergenerational interval of 25 years [17].

\section{Statistical analysis}

Differences between GBA wild-type and mutant enzyme activity were analyzed by the Mann-Whitney $U$ test using SPSS for Windows, version 21.0 (SPSS, Inc., Chicago, IL, USA). Comparisons of height SDS and weight SDS between initial presentation and the latest evaluation were carried out using the Wilcoxon signed-rank test. Values of $p<0.05$ were considered statistically significant.

\section{Supplementary information}

Supplementary information accompanies this paper at https://doi. org/10.1186/s13023-020-01597-0.

Additional file 1: Figure S1. Three-dimensional structure of GBA and location of GBAmutations.

Additional file 2: Table S1. The list of 11 short tandem repeat (STR) markers.

Additional file 3: Table S2. Haplotype analysis of individuals with mutations in the GBAgene.

\section{Abbreviations}

AVN: Avascular necrosis; ERT: Enzyme replacement therapy; GBA: Glucocerebrosidase; GD: Gaucher disease; PCR: Polymerase chain reaction; SDS: Standard deviation score; STR: Short tandem repeat; SNP: Single-nucleotide polymorphism.

\section{Acknowledgements}

We thank the patients enrolled in this study and their parents.

\section{Authors' contributions}

Y-MK and H-WY conceived and designed the study. S-HH and G-HK performed the experiments. Y-MK, H-WY, J-HC, BHL, YBS, JMK, HHL, and CKC analyzed and interpreted the data. Y-MK, J-HC, and H-WY contributed to drafting the manuscript, critical review of the paper, and the approval of the submitted paper. All authors read and approved the final manuscript.

\section{Funding}

This study was supported by research funds from Chungnam National University (2018-0625-01).

\section{Availability of data and materials}

All data generated or analyzed during this study are included in this published article and its supplementary information files.

\section{Ethics approval and consent to participate}

Informed consent was obtained from all patients and their parents, and this study was approved by the Institutional Research Board of each institute. Chungnam National University Hospital IRB No. 2018-07-012. Asan Medical Center IRB No. 2018-0768. Ajou University Hospital IRB No. AJIRB-MED-18-364.
Consent for publication

Not applicable.

\section{Competing interests}

The authors declare that they have no competing interests.

\section{Author details}

${ }^{1}$ Department of Pediatrics, College of Medicine, Chungnam National University, Chungnam National University Sejong Hospital, Sejong, Korea. ${ }^{2}$ Department of Pediatrics, College of Medicine, Chungnam National University, Chungnam National University Hospital, Daejeon, Korea. ${ }^{3}$ Department of Pediatrics, Asan Medical Center Children's Hospital, University of Ulsan College of Medicine, 88 Olympic-ro 43-gil, Songpa-Gu, Seoul 05505, Korea. ${ }^{4}$ Medical Genetics Center, Asan Medical Center, University of Ulsan College of Medicine, Seoul, Korea. ${ }^{5}$ Department of Medical Genetics, Ajou University School of Medicine, Ajou University Hospital, Suwon, Korea. ${ }^{6}$ Department of Pediatrics, College of Medicine, Seoul National University Children's Hospital, Seoul, Korea. ${ }^{7}$ Department of Pediatrics, College of Medicine, Pusan National University Children's Hospital, Yangsan, Korea.

Received: 11 July 2020 Accepted: 26 October 2020

Published online: 11 November 2020

\section{References}

1. Grabowski GA. Phenotype, diagnosis, and treatment of Gaucher's disease. Lancet. 2008;372:1263-71.

2. Nalysnyk L, Rotella P, Simeone JC, Hamed A, Weinreb N. Gaucher disease epidemiology and natural history: a comprehensive review of the literature. Hematology. 2017;22:65-73.

3. Schiffmann R, Sevigny J, Rolfs A, Davies EH, Goker-Alpan O, Abdelwahab $M$, et al. The definition of neuronopathic Gaucher disease. J Inherit Metab Dis. 2020;43:1056-9.

4. Jeong SY, Park SJ, Kim HJ. Clinical and genetic characteristics of Korean patients with Gaucher disease. Blood Cells Mol Dis. 2011:46:11-4.

5. Ida H, Iwasawa K, Kawame H, Rennert OM, Maekawa K, Eto Y. Characteristics of gene mutations among 32 unrelated Japanese Gaucher disease patients: absence of the common Jewish $84 \mathrm{GG}$ and $1226 \mathrm{G}$ mutations. Hum Genet. 1995:95:717-20.

6. Baris HN, Cohen IJ, Mistry PK. Gaucher disease: the metabolic defect, pathophysiology, phenotypes, and natural history. Pediatr Endocrinol Rev. 2014;12:72-81.

7. Dandana A, Ben Khelifa S, Chahed H, Miled A, Ferchichi S. Gaucher disease: clinical, biological and therapeutic aspects. Pathobiology. 2016:83:13-23.

8. Charrow J, Andersson HC, Kaplan P, Kolodny EH, Mistry P, Pastores G, et al. The Gaucher registry: demographics and disease characteristics of 1698 patients with Gaucher disease. Arch Intern Med. 2000;160:2835-43.

9. Lee JY, Lee BH, Kim GH, Jung CW, Lee J, Choi JH, et al. Clinical and genetic characteristics of Gaucher disease according to phenotypic subgroups. Korean J Pediatr. 2012;55:48-53.

10. Choy FY, Zhang W, Shi HP, Zay A, Campbell T, Tang N, et al. Gaucher disease among Chinese patients: review on genotype/phenotype correlation from 29 patients and identification of novel and rare alleles. Blood Cells Mol Dis. 2007;38:287-93.

11. Kim JW, Liou BB, Lai MY, Ponce E, Grabowski GA. Gaucher disease: identification of three new mutations in the Korean and Chinese (Taiwanese) populations. Hum Mutat. 1996;7:214-8.

12. Diaz GA, Gelb BD, Risch N, Nygaard TG, Frisch A, Cohen IJ, et al. Gaucher disease: the origins of the Ashkenazi Jewish N370S and 84GG acid betaglucosidase mutations. Am J Hum Genet. 2000;66:1821-32.

13. Dahl N, Hillborg PO, Olofsson A. Gaucher disease (Norrbottnian type III): probable founders identified by genealogical and molecular studies. Hum Genet. 1993;92:513-5.

14. Ruskey JA, Zhou S, Santiago R, Franche LA, Alam A, Roncière L, et al. The GBA p.Trp378Gly mutation is a probable French-Canadian founder mutation causing Gaucher disease and synucleinopathies. Clin Genet. 2018;94:339-45. 
15. Chaves RG, Pereira Lda V, de Araújo FT, Rozenberg R, Carvalho MD, Coelho $J$, et al. Consanguinity and founder effect for Gaucher disease mutation G377S in a population from Tabuleiro do Norte, Northeastern Brazil. Clin Genet. 2015;88:391-5.

16. Sheth J, Bhavsar R, Mistri M, Pancholi D, Bavdekar A, Dalal A, et al. Gaucher disease: single gene molecular characterization of one-hundred Indian patients reveals novel variants and the most prevalent mutation. BMC Med Genet. 2019;20:31. https://doi.org/10.1186/s12881-019-0759-1.

17. Slatkin M, Rannala B. Estimating the age of alleles by use of intrallelic variability. Am J Hum Genet. 1997;60:447-58.

18. Dvir H, Harel M, McCarthy AA, Toker L, Silman I, Futerman AH, et al. X-ray structure of human acid-beta-glucosidase, the defective enzyme in Gaucher disease. EMBO Rep. 2003:4:704-9.

19. Grabowski GA, Zimran A, Ida H. Gaucher disease types 1 and 3: Phenotypic characterization of large populations from the ICGG Gaucher Registry. Am J Hematol. 2015;90:S12-8.

20. Koprivica V, Stone DL, Park JK, Callahan M, Frisch A, Cohen IJ, et al. Analy sis and classification of 304 mutant alleles in patients with type 1 and type 3 Gaucher disease. Am J Hum Genet. 2000;66:1777-86.

21. Rosenbloom BE, Weinreb NJ. Gaucher disease: a comprehensive review. Crit Rev Oncog. 2013;18:163-75.

22. Pastores GM, Hughes DA, et al. Gaucher disease. In: Adam MP, Ardinger $\mathrm{HH}$, Pagon RA, et al., editors. GeneReviews ${ }^{\circledR}$. Seattle: University of Washington; 1993.

23. Fairley C, Zimran A, Phillips M, Cizmarik M, Yee J, Weinreb N, et al. Phenotypic heterogeneity of N370S homozygotes with type I Gaucher disease: an analysis of 798 patients from the ICGG Gaucher Registry. J Inherit Metab Dis. 2008;31:738-44.

24. Zhao H, Bailey LA, Elsas $\sqcup$, Grinzaid KA, Grabowski GA. Gaucher disease: in vivo evidence for allele dose leading to neuronopathic and nonneuronopathic phenotypes. Am J Hum Genet. 2003;116A:52-6.

25. Germain DP, Puech JP, Caillaud C, Kahn A, Poenaru L. Exhaustive screening of the acid beta-glucosidase gene, by fluorescence-assisted mismatch analysis using universal primers: mutation profile and genotype/phenotype correlations in Gaucher disease. Am J Hum Genet. 1998:63:415-27.

26. Park JK, Orvisky E, Tayebi N, Kaneski C, Lamarca ME, Stubblefield BK, et al. Myoclonic epilepsy in Gaucher disease: genotype-phenotype insights from a rare patient subgroup. Pediatr Res. 2003;53:387-95.

27. Hruska KS, LaMarca ME, Scott CR, Sidransky E. Gaucher disease: mutation and polymorphism spectrum in the glucocerebrosidase gene (GBA). Hum Mutat. 2008;29:567-83.

28. Tylki-Szymańska A, Vellodi A, El-Beshlawy A, Cole JA, Kolodny E. Neuronopathic Gaucher disease: demographic and clinical features of 131 patients enrolled in the International Collaborative Gaucher Group Neurological Outcomes Subregistry. J Inherit Metab Dis. 2010;33:339-46.

29. Kraoua I, Sedel F, Caillaud C, Froissart R, Stirnemann J, Chaurand G, et al. A French experience of type 3 Gaucher disease: Phenotypic diversity and neurological outcome of 10 patients. Brain Dev. 2011;33:131-9.

30. Nagappa M, Bindu PS, Taly AB, Sinha S. Oculomotor apraxia in Gaucher disease. Pediatr Neurol. 2015;52:468-9.

31. Choi JM, Kim WC, Lyoo CH, Kang SY, Lee PH, Baik JS, et al. Association of mutations in the glucocerebrosidase gene with Parkinson disease in a Korean population. Neurosci Lett. 2012;514:12-5.

32. Do J, McKinney C, Sharma P, Sidransky E. Glucocerebrosidase and its relevance to Parkinson disease. Mol Neurodegener. 2019;14:36. https:// doi.org/10.1186/s13024-019-0336-2.

33. Kang E, Kim YM, Kim GH, Lee BH, Yoo HW, Choi JH. Mutation spectrum of STAR and a founder effect of the p.Q258* in Korean patients with congenital lipoid adrenal hyperplasia. Mol Med. 2017;23:149-54.

34. Kim SY, Kim AR, Kim NK, Kim MY, Jeon EH, Kim BJ, et al. Strong founder effect of pP240L in CDH23 in Koreans and its significant contribution to severe-to-profound nonsyndromic hearing loss in a Korean pediatric population. J Transl Med. 2015;13:263. https://doi.org/10.1186/s1296 7-015-0624-8.

35. Seo JY, Song JS, Lee KO, Won HH, Kim JW, Kim SH, et al. Founder effects in two predominant intronic mutations of UNC13D, c.118-308C > T and C.754-1G >C underlie the unusual predominance of type 3 familial hemophagocytic lymphohistiocytosis (FHL3) in Korea. Ann Hematol. 2013;92:357-64.

36. Kim YM, Choi JH, Lee BH, Kim GH, Kim KM, Yoo HW. Predominance of the c.648G > T G6PC gene mutation and late complications in Korean patients with glycogen storage disease type la. Orphanet J Rare Dis. 2020;15:45. https://doi.org/10.1186/s13023-020-1321-0.

37. Ferenci $P$ (2006) Regional distribution of mutations of the ATP7B gene in patients with Wilson disease: impact on genetic on genetic testing. Hum Genet 120:151-159

38. Jeong SY, Kim SJ, Yang JA, Hong JH, Lee SJ, Kim HJ. Identification of a novel recombinant mutation in Korean patients with Gaucher disease using a long-range PCR approach. J Hum Genet. 2011;56:469-71.

\section{Publisher's Note}

Springer Nature remains neutral with regard to jurisdictional claims in published maps and institutional affiliations.
Ready to submit your research? Choose BMC and benefit from:

- fast, convenient online submission

- thorough peer review by experienced researchers in your field

- rapid publication on acceptance

- support for research data, including large and complex data types

- gold Open Access which fosters wider collaboration and increased citations

- maximum visibility for your research: over 100M website views per year

At BMC, research is always in progress.

Learn more biomedcentral.com/submissions 